\title{
Roedores muroideos (Mammalia, Cricetidae e Muridae) da Toca dos Ossos, Ourolândia, Caatinga do estado da Bahia, Brasil \\ Muroid rodents (Mammalia, Cricetidae and Muridae) from Toca dos Ossos, Ourolândia, Caatinga of the State of Bahia, Brazil
}

\author{
Anny Caroliny Gomes (D) Carolina Saldanha Schererl (D) Patrícia Hadler" (1)

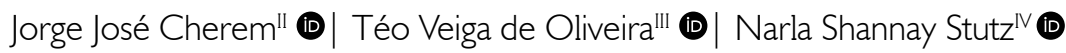 \\ 'Universidade Federal do Recôncavo da Bahia. Cruz das Almas, Bahia, Brasil \\ "Universidade Federal de Santa Catarina. Florianópolis, Santa Catarina, Brasil \\ "'Universidade Estadual de Feira de Santana. Feira de Santana, Bahia, Brasil \\ wUniversidade Federal do Rio Grande do Sul. Porto Alegre, Rio Grande do Sul, Brasil
}

\begin{abstract}
Resumo: Roedores muroideos incluem as duas familias mais diversas de mamíferos do mundo, Cricetidae e Muridae. Apesar disso, são ainda pouco estudados em muitas regiões, como a Caatinga. O presente estudo dá a conhecer os roedores muroideos da Toca dos Ossos, situada em Ourolândia, centro-norte da Bahia. A Toca dos Ossos é uma cavidade natural escavada em maciço calcário da Formação Caatinga, destacando-se por ser um importante sítio paleontológico. O material estudado é constituído por fragmentos cranianos e mandibulares coletados em duas áreas da caverna. Em uma delas, egagropilos de Tyto furcata foram coletados em uma dolina e, na outra, o material foi coletado no sedimento de superfície em salões e condutos internos da caverna. Sete espécies foram registradas: Necromys lasiurus, a mais frequente na amostra, Cerradomys sp., Holochilus sciureus, Oligoryzomys sp., Calomys sp., Wiedomys pyrrhorhinos e Rattus rattus, este exclusivo nas amostras de egagropilos. Apesar das diferenças nas frequências dos táxons, as amostras em ambas as áreas da caverna apresentam grande similaridade taxonômica e são compostas por espécies de ocorrência atual na Caatinga, incluindo a espécie exótica $R$. rattus, o que sugere uma idade recente para o material, posterior à expansão desse bioma, que ocorreu há cerca de 4.000 anos.
\end{abstract}

Palavras-chave: Caverna. Egagropilo de coruja. Floresta tropical sazonal seca. Sigmodontinae.

Abstract: Muroid rodents include the two most diversified mammalian families of the world, Cricetidae and Muridae. Despite this, muroids are still poorly addressed in many regions, such as the Caatinga biome. This study reports the muroid rodents from the 'Toca dos Ossos', municipality of Ourolândia, central-northern Bahia state. The 'Toca dos Ossos' is a natural cavity excavated in the limestone massif of the Caatinga Formation, standing out as an important paleontological site. The studied material consists of cranial and mandibular fragments retrieved in two cave areas. One of them represent Tyto furcata pellets collected at a sinkhole, and the other is from material collected in the surface sediment of cave halls and internal conduits. The following species were recorded: Necromys lasiurus, the most frequent in the samples, Cerradomys sp., Holochilus sciureus, Oligoryzomys sp., Calomys sp., Wiedomys pyrrhorhinos, and Rattus rattus, the latter exclusively found in the owl pellets samples. Even though there are differences in the frequencies of the taxa, the communities from both of the cave areas possess great taxonomic similarity and include species currently found in the Caatinga, including the exotic species $R$. rattus, suggesting a recent age for the material, subsequent to the expansion of this biome, which happened 4,000 years ago.

Keywords: Cave. Owl pellet. Seasonally dry tropical forest. Sigmodontinae.

GOMES, A. C., C. S. SCHERER, P. HADLER, J. J. CHEREM, T. V. OLIVEIRA \& N. S. STUTZ, 2020. Roedores muroideos (Mammalia, Cricetidae e Muridae) da Toca dos Ossos, Ourolândia, Caatinga do estado da Bahia, Brasil. Boletim do Museu Paraense Emílio Goeldi. Ciências Naturais 15(3): 777-794. DOI: http://doi.org/10.46357/bcnaturais.v15i3.215.

Autora para correspondência: Anny Caroliny Gomes. Universidade Federal do Recôncavo da Bahia. Campus Cruz das Almas. Centro de Ciências Agrárias, Ambientais e Biológicas. Cruz das Almas, BA, Brasil. CEP 44380-000 (carolina.ss@ufrb.edu.br, annycarolinyf@yahoo.com.br). Recebido em 29/11/2019

Aprovado em 08/10/2020

Responsabilidade editorial: Alexandra Maria Ramos Bezerra
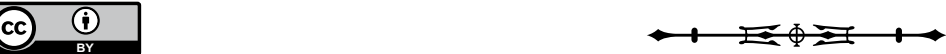


\section{INTRODUÇÃO}

Os muroideos compreendem um grupo de roedores miomorfos caracterizados por sua fórmula dentária simplificada (ausência de pré-molares nas mandíbulas e maxilas) e pela presença de anterocone/ídeo bem desenvolvido no primeiro molar (Flynn et al., 1985). Eles compõem uma parte significativa das assembleias de mamíferos na maioria dos continentes, ocorrem nos mais variados ambientes, perfazendo, aproximadamente, $28 \%$ do total de espécies de mamíferos, e incluem as duas famílias mais diversas de mamíferos, Cricetidae (792 espécies) e Muridae (834 espécies) (Jansa \& Weksler, 2004; Honeycutt et al., 2007; Fabre et al., 2012; Burgin et al., 2018).

NaAmérica do Sul, a família Cricetidae é representada pela subfamília Sigmodontinae, que inclui mais de 430 espécies viventes e ilustra um dos mais extraordinários eventos de radiação explosiva, considerando que sua história evolutiva no continente pode se estender por um período inferior a 4 milhões de anos (Pardiñas et al., 2017; Barbière et al., 2019). Por outro lado, as três espécies de Muridae distribuídas amplamente na América do Sul são invasoras recentes, tendo sido introduzidas acidentalmente pelos exploradores do Novo Mundo (Catzeflis, 2018).

A Caatinga é um bioma típico do semiárido do Nordeste brasileiro, formada por um mosaico de ambientes arbustivos e florestais (Leal et al., 2005). Atualmente considerada uma das maiores áreas de floresta tropical sazonalmente seca do Novo Mundo, a Caatinga abriga uma biodiversidade considerável para uma região semiárida, composta por, pelo menos, 3.150 espécies de plantas, 98 de anfíbios, 79 de répteis e 548 de aves (Silva et al., 2017). Em relação aos mamíferos, Carmignotto \& Astúa (2017) registraram 183 espécies autóctones, 11 das quais são endêmicas do bioma. Rodentia é a segunda ordem em riqueza, com 41 espécies, sendo que 23 delas pertencem à família Cricetidae (Carmignotto \& Astúa, 2017). Além disso, as três espécies de murídeos invasores também estão presentes na Caatinga (Moojen, 1952).
Nesse bioma, destaca-se a Reserva Particular do Patrimônio Natural (RPPN) Toca dos Ossos, criada em 27 de dezembro de 2016 (INEMA, 2016), a qual tem, entre outros aspectos, elevada relevância paleontológica (e.g., Cartelle \& Lessa, 1988, 1989; Cartelle \& Abuhid, 1994; Czaplewski \& Cartelle, 1998; Lessa et al., 1998; Lobo et al., 2017; Gomes et al., 2019). Por outro lado, poucos estudos foram realizados sobre a fauna atual de mamíferos da Toca dos Ossos e região do entorno (e.g., Sbragia \& Cardoso, 2008; Jesus \& Oliveira, 2017). Entre os roedores da Toca dos Ossos, foram conduzidos apenas estudos sobre variação em caracteres cranianos do caviomorfo Kerodon rupestris (WiedNeuwied, 1820) (Lessa \& Pessôa, 2005; Lessa et al., 2005).

Apesar da elevada riqueza dos roedores no estado da Bahia como um todo, levantamentos abordando esse grupo têm sido conduzidos principalmente na Mata Atlântica (e.g., Vaz, 2005; Geise \& Pereira, 2008; Carvalho \& Oliveira, 2015), havendo, entretanto, poucos na Caatinga (Oliveira et al., 2003; Freitas et al., 2005; Oliveira \& Pessôa, 2005; Pereira \& Geise, 2009). Devido à escassez de registros de roedores neste bioma, é de fundamental importância o estudo da fauna da Toca dos Ossos. Neste sentido, este trabalho tem como objetivos fornecer uma lista comentada de roedores muroideos dessa localidade, bem como apontar o provável intervalo temporal de depósito do material.

\section{MATERIAL E MÉTODOS}

\section{ÁREA DE ESTUDO}

A Toca dos Ossos está localizada na bacia do rio Salitre, município de Ourolândia, estado da Bahia, região Nordeste do Brasil. Sua entrada principal situa-se $560 \mathrm{~m}$ acima do nível do mar (1055' 51,25”' S, 41 3' 27,10" O) (Figuras 1A e 1B). A Toca dos Ossos representa uma cavidade natural escavada em um maciço calcário da Formação Caatinga, situada na porção norte da Chapada Diamantina, com grande concentração de fósseis (e.g., Lessa et al., 1998; Gomes et al., 2019). 
A Toca dos Ossos possui 14.200 m de projeção horizontal mapeados (Rubbioli et al., 2019). Apresenta padrões morfológicos peculiares que a diferem de outras cavernas brasileiras, com galerias meandrantes interconectadas em vários pontos. Entre as galerias maiores, pode ser observada uma complexa rede labiríntica, responsável pela ligação de áreas distintas da caverna (Figura 1C) (Auler et al., 2006; Rubbioli et al., 2019). Esse aspecto espongiforme, sem padrões definidos, fica evidente no período chuvoso, quando o alagamento da galeria principal escoa água para as áreas mais baixas da caverna, enquanto as áreas posicionadas em um nível superior permanecem com seu sedimento seco (Auler et al., 2006).

A bacia do rio Salitre, na qual se situa a Toca dos Ossos, está inserida no bioma Caatinga e é caracterizada predominantemente por duas fitofisionomias, Caatinga Arbustiva (esparsa ou densa, formada por arbustos de 2 a 3 metros de altura e arvoretas de 5 a 6 metros) e Caatinga Arbórea (composta predominantemente por árvores de 8 a 10 metros) (Rizzini, 1997; CBHS, 2017). No entanto, grande parte da vegetação nessa região encontra-se atualmente alterada por ação antrópica, com a conversão de quase 50\% da cobertura vegetal natural em áreas de cultivo agrícola e pastagem. As estações seca e chuvosa são bem definidas, e a estação chuvosa está concentrada nos meses de novembro a março. A precipitação total anual varia de 300 a 600 mm (CBHS, 2017). O clima, segundo a classificação de Köppen, é do tipo BSh, semiárido, de baixas latitude e altitude, caracterizado pela baixa pluviosidade e temperatura média anual de cerca de $25^{\circ} \mathrm{C}$ (Alvares et al., 2013).

\section{AMOSTRAGEM}

O material estudado consiste de 210 fragmentos cranianos e mandibulares, coletados em 03 de julho de 2011, 10-11 de agosto de 2012 e 09 de junho de 2013, em duas áreas da Toca dos Ossos (doravante denominadas TO), distantes 500 metros uma da outra, aproximadamente. A primeira compreende uma dolina, denominada TO-I (Figuras 1C e 1D), onde o material foi obtido a partir da triagem de egagropilos inteiros ou desagregados de corujas-das-igrejas - Tyto furcata (Temminck, 1827) - (Figura 1E). A segunda área, denominada TO-II, localiza-se na parte mais interna da caverna e está composta por vários salões e condutos (Figuras $1 \mathrm{C} \mathrm{e} \mathrm{1F),} \mathrm{onde} \mathrm{o} \mathrm{material} \mathrm{foi} \mathrm{coletado} \mathrm{diretamente}$ do sedimento de superfície (Figura 1G). A coleta dos espécimes na caverna foi autorizada pelo Instituto Chico Mendes de Conservação da Biodiversidade (ICMBio), por meio das licenças números 27530 e 35904.

\section{TRIAGEM E IDENTIFICAÇÃO}

Em laboratório, o material foi limpo sob estereomicroscópio binocular, com o auxílio de pincel e agulha. Posteriormente, os espécimes da TO-I foram depositados na Coleção de Mamíferos do Museu de Zoologia da Universidade Estadual de Feira de Santana (MZFS-DM) e da TO-II nas coleções Paleontologia de Vertebrados do Museu de Zoologia e Paleontologia da Universidade Federal do Recôncavo da Bahia (UFRB-PV).

A identificação das espécies foi realizada a partir de fontes bibliográficas (Pardiñas \& Teta, 2011; Bonvicino, 2015; Brandão \& Nascimento, 2015; Salazar-Bravo, 2015; Weksler \& Bonvicino, 2015; Catzeflis, 2018; Cherem et al., 2018; Stutz et al., 2018) e da comparação com espécimes depositados nas coleções de mamíferos do Museu de Zoologia da Universidade Estadual de Feira de Santana (MZFS-DM) e do Departamento de Ecologia e Zoologia da Universidade Federal de Santa Catarina (UFSC) (Apêndice 1). Quando necessário, foram tomadas medidas utilizando-se paquímetro digital com precisão de 0,02 mm: largura do forâmen incisivo (LFI); comprimento da série molar superior (CM1-M3); comprimento da série molar inferior (Cm1-m3); comprimento do primeiro molar inferior ( $\mathrm{Cm} 1)$.

A nomenclatura dentária seguiu Reig (1977) e Percequillo (2006). A nomenclatura e o ordenamento taxonômicos seguiram Patton et al. (2015) para Sigmodontinae e Wilson \& Reeder (2005) para Muridae. O número mínimo de indivíduos (NMI) por espécie foi determinado segundo Beisaw (2013). 
A

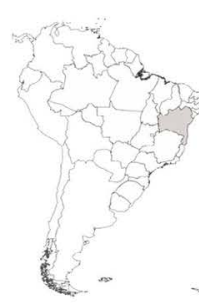

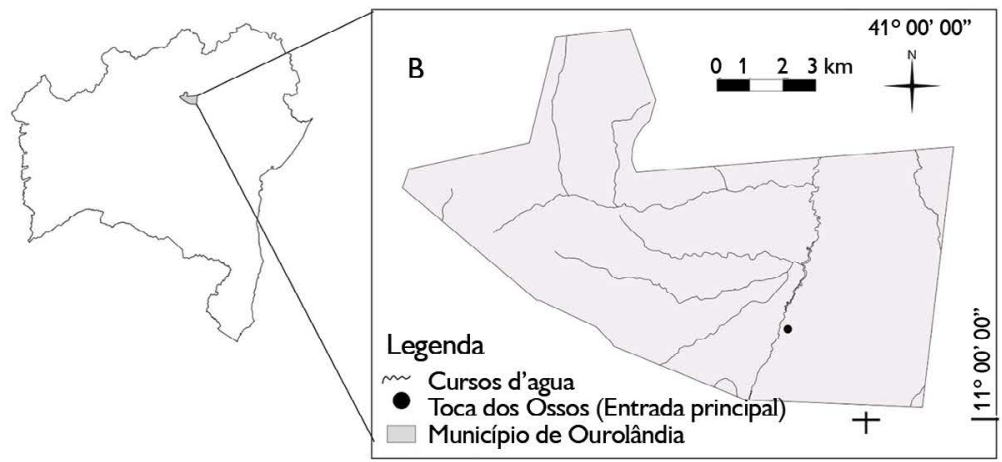
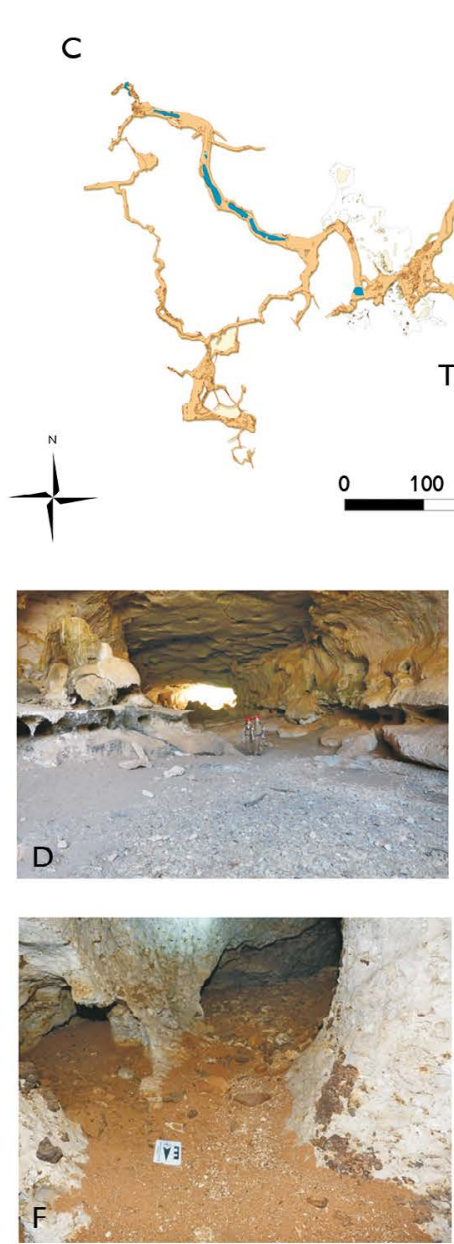
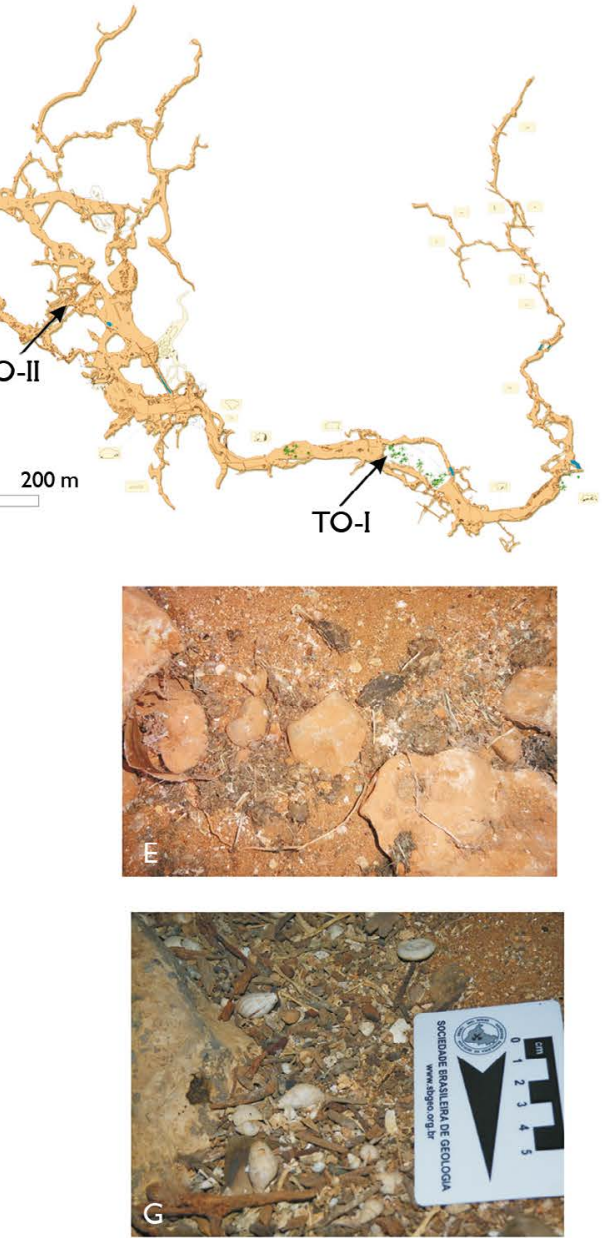

Figura 1. Localização e imagens da Toca dos Ossos: A) mapa da América do Sul, com destaque para o estado da Bahia, no Brasil; B) município de Ourolândia; C) mapa da caverna Toca dos Ossos, indicando os pontos de coleta do material (modificado de Rubbioli et al., 2019); D) dolina da Toca dos Ossos-I; E) egagropilos de Tyto furcata coletados na Toca dos Ossos-I; F) porção interna da caverna, denominada Toca dos Ossos-II; G) detalhe do material coletado no sedimento de superfície da Toca dos Ossos-II. Mapas: modificados de Rubbioli et al. (2019). Fotos: C. S. Scherer (2013).

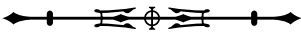




\section{RESULTADOS}

\section{COMPOSIÇÃO DAS AMOSTRAS}

A amostra total de roedores muroideos da Toca dos Ossos foi composta por duas famílias e sete espécies, com NMI = 99 (Tabela 1; Apêndice 2). Entre os cricetídeos, foram registrados táxons pertencentes a quatro tribos: Akodontini (33,3\% dos indivíduos), que apresentou a maior abundância relativa, e Phyllotini (22,2\%), Wiedomyini (21,2\%) e Oryzomyini (21,3\%), com abundâncias similares. Apesar de menos frequente na amostra, a tribo Oryzomyini apresentou maior riqueza, com três táxons (Cerradomys sp., Holochilus sciureus Wagner, 1842 e Oligoryzomys sp.). Rattus rattus (2\%) foi o único representante da familia Muridae identificado na área de estudo.

A amostra TO-I apresentou NMI $=39$ e foi representada por seis espécies de Cricetidae e uma de Muridae. A amostra TO-II, com NMI $=60$, incluiu as mesmas seis espécies de Cricetidae. Apesar da composição taxonômica semelhante, as duas amostras apresentaram diferenças nas frequências dos táxons. Necromys lasiurus
(Lund, 1840) foi o táxon dominante $(56,4 \%)$ em TO-I, seguido por Wiedomys pyrrhorhinos (Wied-Neuwied, 1821) (18\%) e Calomys sp. (10,4\%). Por outro lado, Calomys sp. predominou na TO-II, com 30\%, seguido por $W$. pyrrhorhinos, com 23,3\%, N. lasiurus e H. sciureus, ambos com 18,3\% (Tabela 1).

\section{IDENTIFICAÇÃO DOS TÁXONS}

Família Cricetidae Fischer, 1817

Subfamília Sigmodontinae Wagner, 1843

Tribo Akodontini Vorontsov, 1959

Necromys lasiurus (Lund, 1840)

Figuras 2A, 2B, 3A, 4A e 5A

Material: 18 crânios, 21 maxilares e 39 dentários (Apêndice 2).

Descrição: material de médio porte. Crânio alto com rostro curto e forâmen incisivo longo, estendendose posteriormente até o nível do protocone do M1; crista supraorbital presente, mas pouco marcada. Dentário baixo e robusto, com projeção capsular desenvolvida.

Tabela 1. Número mínimo de indivíduos (NMI) e percentual de indivíduos por táxon registrado nas duas áreas de amostragem (TO-I e TO-II) na Toca dos Ossos, Ourolândia, estado da Bahia, Brasil.

\begin{tabular}{|c|c|c|c|c|c|c|}
\hline \multirow{2}{*}{ Táxon } & \multicolumn{2}{|c|}{ TO-I } & \multicolumn{2}{|c|}{ TO-II } & \multicolumn{2}{|c|}{ Total } \\
\hline & NMI & $\%$ & NMI & $\%$ & NMI & $\%$ \\
\hline \multicolumn{7}{|l|}{ Família Cricetidae } \\
\hline \multicolumn{7}{|l|}{ Tribo Akodontini } \\
\hline Necromys lasiurus (Lund, 1840) & 22 & 56,4 & 11 & 18,3 & 33 & 33,3 \\
\hline \multicolumn{7}{|l|}{ Tribo Oryzomyini } \\
\hline Cerradomys sp. & 1 & 2,5 & 5 & 8,4 & 6 & 6,1 \\
\hline Holochilus sciureus Wagner, 1842 & 2 & 5,1 & 11 & 18,3 & 13 & 13,2 \\
\hline Oligoryzomys sp. & 1 & 2,5 & 1 & 1,7 & 2 & 2,0 \\
\hline \multicolumn{7}{|l|}{ Tribo Phyllotini } \\
\hline Calomys sp. & 4 & 10,4 & 18 & 30 & 22 & 22,2 \\
\hline \multicolumn{7}{|l|}{ Tribo Wiedomyini } \\
\hline Wiedomys pyrrhorhinos (Wied-Neuwied, 1821) & 7 & 18 & 14 & 23,3 & 21 & 21,2 \\
\hline \multicolumn{7}{|l|}{ Família Muridae } \\
\hline Rattus rattus (Linnaeus, 1758) & 2 & 5,1 & 0 & 0 & 2 & 2,0 \\
\hline Total & 39 & 100 & 60 & 100 & 99 & 100 \\
\hline
\end{tabular}

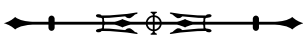


Molares com cúspides alternadas; flexo anteromediano ausente; mesolofo ausente, exceto em UFRB-PV 2857, 2975 e MZFS-DM 0820, 0864; flexídeo anteromediano presente apenas em espécimes jovens (UFRB-PV 2998, 3002, 3031, 3119; MZFS-DM0841, 0843, 0847, 0848, 0855, 0861, 0862, 0863, 0874 e 0875); mesolofídeo ausente.

Comentários: as características apresentadas, em particular sobre seu porte, desenvolvimento do rostro, extensão do forâmen incisivo, presença de crista supraorbital e morfologia dos molares, permitiram atribuir esses espécimes a N. lasiurus, de acordo com Anderson \& Olds (1989), Pardiñas et al. (2015b) e Stutz et al. (2018). Necromys lasiurus foi o roedor mais frequente na Toca dos Ossos e único representante da tribo Akodontini registrado nas amostras. Carmignotto \& Astúa (2017) relacionaram uma segunda espécie da tribo para a Caatinga, Akodon cursor (Winge, 1887), que se diferencia de $N$. lasiurus principalmente por seu crânio mais baixo e crista supraorbital ausente (Geise, 2012). Além disso, Akodon Meyen, 1833 apresenta flexo/ídeo anteromediano e mesolofo/ídeo (exceto em espécimes mais velhos, com molares muito gastos) (Stutz et al., 2018).

\section{Tribo Oryzomyini Vorontsov, 1959}

Cerradomys sp.

Figuras 3B, 4B e 5B

Material: um crânio fragmentado, seis maxilares e seis dentários (Apêndice 2).

Descrição: material de médio porte. Forâmen incisivo, geralmente se estende posteriormente até a margem anterior do M1; palato longo, estendendo-se posteriormente à margem posterior do $M 3$, exceto em espécimes jovens (UFRB-PV 959, 2820 e 2990). Dentário alto e robusto, com projeção capsular bem desenvolvida; processo coronoide pequeno; crista massetérica inferior pouco marcada. Molares pentalofodontes; M2 com paracone e protocone opostos; flexo/ídeo anteromediano ausente; cíngulo anterolabial presente nos molares inferiores; procíngulo no $\mathrm{m} 1$ curto; mesolofídeo bem desenvolvido.
Comentários: o tamanho e a morfologia dos molares, conforme descrito, são caracteres diagnósticos do gênero Cerradomys Weksler, Percequillo \& Voss, 2006, de acordo com Weksler et al. (2006) e Percequillo et al. (2008). Em relação ao material utilizado para comparação (Apêndice 1), Cerradomys apresenta forâmen incisivo mais longo, projeção capsular mais desenvolvida e hipoflexídeo menos profundo do que Hylaeamys Weksler, Percequillo \& Voss, 2006; palato mais longo do que Rhipidomys Coues, 1884, o qual apresenta flexo/ídeo anteromediano; crista massetérica inferior menos marcada e molares pouco maiores do que em Euryoryzomys Weksler, Percequillo \& Voss, 2006; forâmen incisivo mais longo, dentário mais alto e diastema mais longo do que em Oecomys Thomas, 1906. O dentário mais robusto, mas com processo coronoide mais baixo, a posição relativa do protocone e paracone no M2 e a presença de mesolofídeo nos molares inferiores permitem diferenciar Cerradomys de Wiedomys Hershkovitz, 1959.

Cerradomys inclui oito espécies, duas das quais estão presentes atualmente na Caatinga, Cerradomys langguthi Percequillo, Hingst-Zaher \& Bonvicino, 2008 e Cerradomys vivoi Percequillo, Hingst-Zaher \& Bonvicino, 2008 (Bonvicino et al., 2014; Carmignotto \& Astúa, 2017). Dentre essas duas, apenas $C$. vivoi possui registro recente para o estado da Bahia (Percequillo et al., 2008). Os espécimes registrados na Toca dos Ossos foram referidos como Cerradomys sp. por não haver caracteres morfológicos que permitam distinguir confiavelmente essas duas espécies (Percequillo et al., 2008; Caccavo \& Oliveira, 2016).

\section{Holochilus sciureus Wagner, 1842}

Figuras 2C, 2D, 3C, 4C e 5C

Material: quatro crânios, 11 maxilares e nove dentários (Apêndice 2).

Descrição: material de grande porte, o maior entre os cricetídeos nas amostras da Toca dos Ossos. Palato longo, estendendo-se além da margem posterior do M3; forâmen incisivo muito curto, não alcançando a margem anterior do M1. Dentário robusto e crista

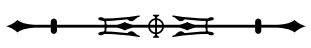


massetérica inferior bem marcada. Molares com cúspides alternadas e lofídeos com margem prismática; fossetídeo anteromediano pequeno; borda posterior do proto e hipoconídeo orientado transversalmente.

Comentários: as características listadas e a ausência de mesolofídeo permitiram identificar o material como H. sciureus, conforme Pardiñas \& Teta (2011) e Brandão \& Nascimento (2015). Esta é a única espécie do gênero com ocorrência atual na Caatinga (Carmignotto \& Astúa, 2017).

Oligoryzomys sp.

Figuras 2E, 2F, 3D, 4D e 5D

Material: um crânio, um maxilar e dois dentários (Apêndice 2).

Descrição: material de tamanho diminuto. Forâmen incisivo estendendo-se posteriormente até o nível da borda anterior do M1. Dentário alto, com dois alvéolos para as raízes do $\mathrm{m} 2$ e m3. Molares baquiodontes; mesolofo presente no M1 e M2; flexo/ídeo anteromediano presente, mas pouco marcado (MZFS-DM 0825) ou perdido pelo desgaste (UFRB-PV 2735, MZFS-DM 0856); mesoestilídeo presente no $\mathrm{m} 2$, mas mesolofídeo ausente.

Comentários: entre os espécimes da Toca dos Ossos, Oligoryzomys sp. Bangs, 1900 apresenta porte similar a Calomys sp., diferenciando-se pela menor extensão posterior do forâmen incisivo, menor número de alvéolos no M2/m2 e M3/m3 (três nesses molares de Calomys), e presença de mesolofo.

Quatro espécies de Oligoryzomys Bangs, 1900 ocorrem atualmente na Caatinga (Carmignotto \& Astúa, 2017). Entre elas, O. rupestris Weksler \& Bonvicino, 2005 apresenta o anterocone não dividido por um flexo anteromediano (Weksler \& Bonvicino, 2015), diferindo do material da Toca dos Ossos. Oligoryzomys nigripes (Olfers, 1818) e O. stramineus Bonvicino \& Weksler, 1998 apresentam porte relativamente grande para o gênero, enquanto O. mattogrossae (Allen, 1916) é comparativamente menor (Weksler et al., 2017), mas há sobreposição nas medidas dentárias entre essas espécies (Tabela 2). Desta forma, considerando o pequeno tamanho da amostra da Toca dos Ossos, sua natureza fragmentária e a ausência de caracteres claramente diagnósticos, esses espécimes foram identificados apenas em nível de gênero.

Tribo Phyllotini Vorontsov, 1959

Calomys sp.

Figuras 2G, 2H, 3E, 4E e 5E

Material: seis crânios, nove maxilares e 36 dentários (Apêndice 2).

Tabela 2. Comparação entre as medidas dentárias (em milímetros) dos espécimes de Oligoryzomys sp. registrados na Toca dos Ossos (MZFS-DM 0825 e UFRB-PV 2735) e das espécies do gênero presentes na Caatinga: 1 = espécimes de O. mattogrossae do Nordeste do Brasil (Weksler et al., 2017); 2 = espécimes de O. mattogrossae ao longo de toda a distribuição (Weksler et al., 2017$) ; 3$ = espécimes de $O$. rupestris, $O$. stramineus e 0 . nigripes ao longo de suas distribuições (Weksler \& Bonvicino, 2005); $4=$ espécimes de 0 . nigripes do estado do Espírito Santo ao estado do Rio Grande do Sul (Machado et al., 2011); n = número de espécimes.

\begin{tabular}{|c|c|c|}
\hline Espécimes/Espécies & Comprimento M1-M3 & Largura M1 \\
\hline MZFS-DM 0825 & 3,37 & 1,05 \\
\hline UFRB-PV 2735 & - & 1,00 \\
\hline O. mattogrossae ${ }^{1}$ & $2,94-3,15(n=4)$ & $0,86-0,96(n=4)$ \\
\hline O. mattogrossae ${ }^{2}$ & $2,84-3,64(n=39)$ & $0,81-1,20(n=39)$ \\
\hline O. rupestris ${ }^{3}$ & $3,2-3,5(n=8)$ & $1,0-1,1(n=8)$ \\
\hline O. stramineus ${ }^{3}$ & $3,3-4,2(n=36)$ & $1,0-1,3(n=36)$ \\
\hline O. nigripes $^{3}$ & $3,5-4,0(n=35)$ & $1,0-1,2(n=35)$ \\
\hline O. nigripes ${ }^{4}$ & $3,06-4,28(n=153)$ & $0,84-1,12(n=155)$ \\
\hline
\end{tabular}

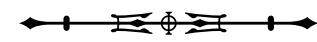


Descrição: espécimes de tamanho diminuto. Crânio com crista supraorbital desenvolvida e forâmen incisivo longo, alcançando o protocone do M1. Dentário delicado, mas alto; projeção capsular medianamente desenvolvida; três alvéolos para as raízes do M2/m2 e $\mathrm{M} 3 / \mathrm{m} 3$. Molares tetralofodontes e braquiodontes; M1 com flexo anteromediano muito profundo, dividindo o anterocone em dois cônulos, sendo um menor, anterolingual, e outro maior, anterolabial. No espécime UFRB-PV 2991, o flexo anteromediano não está visível, em razão do desgaste.

Comentários: o material foi atribuído ao gênero Calomys pelo conjunto de características descritas, conforme Salazar-Bravo (2015). Calomys foi o único representante da tribo Phyllotini registrado nas amostras. Revisões recentes têm apontado C. expulsus (Lund, 1840) como a única espécie do gênero presente na Caatinga (Salazar-Bravo, 2015; Carmignotto \& Astúa, 2017). No entanto, considerando a natureza fragmentária dos espécimes da Toca dos Ossos e a ausência de caracteres diagnósticos que permitam a identificação da espécie, optou-se por tratar o material como Calomys sp.

Tribo Wiedomyini Reig, 1980

Wiedomys pyrrhorhinos (Wied-Neuwied, 1821)

Figuras 2I, 2], 3F, 4F e 5F

Material: 14 crânios, 12 maxilares e 12 dentários (Apêndice 2).

Descrição: material de tamanho mediano nas amostras da Toca dos Ossos. Palato curto, não se estendendo além da margem posterior do M3, exceto em três espécimes (MZFS-DM 0956, 0965 e 2907), nos quais o palato é longo; forâmen incisivo longo, com sua borda posterior ultrapassando a margem anterior do M1. Dentário comparativamente delicado e processo coronoide baixo. M2 com paracone e protocone alternados; flexo/ídeo anteromediano conspícuo, exceto em indivíduos com maior desgaste (MZFS-DM
0905, 0955, 0956, 0965; UFRB-PV 906, 2819, 2897, 2956, 2957, 2983, 3121 e 3176); mesolofo presente; mesolofídeo ausente; $\mathrm{m} 1$ com procíngulo longo.

Comentários: o comprimento do forâmen incisivo, a alternância das cúspides, a presença de mesolofo e a ausência de mesolofídeo estão entre os caracteres diagnósticos de Wiedomys, assim como o palato curto (Bonvicino, 2015). No entanto, alguns espécimes podem apresentar palato longo, como observado em três casos da Toca dos Ossos e no espécime ilustrado por Bezerra et al. (2013, fig. 2).

Wiedomys foi o único representante da tribo Wiedomyini registrado para a Toca dos Ossos. O gênero inclui duas espécies, $W$. pyrrhorhinos e $W$. cerradensis Gonçalves, Almeida \& Bonvicino, 2005, ambas com ocorrência atual na Caatinga (Carmignotto \& Astúa, 2017). De acordo com Bonvicino (2015), W. pyrrhorhinos apresenta forâmen incisivo mais largo $(>2,22 \mathrm{~mm})$ e série molar superior mais longa (> 4,6 mm) do que W. cerradensis. Desta maneira, as medidas do material da Toca dos Ossos - LFI = 2,30-2,57 mm ( $\mathrm{n}=12)$; CM1-M3 $=4,56-4,81 \mathrm{~mm}(\mathrm{n}=17)$ - indicam se tratar de W. pyrrhorhinos.

Família Muridae Illiger, 1811

Rattus rattus (Linnaeus, 1758)

Figura 3G

Material: dois dentários (Apêndice 2).

Descrição: espécimes de grande porte. Dentários grandes e robustos, com projeção capsular pouco marcada e processo coronoide desenvolvido.

Comentários: a família Muridae está representada no continente americano por dois gêneros: Mus inclui apenas uma espécie, M. musculus (Linnaeus, 1758), de pequeno porte, enquanto Rattus Fischer de Waldheim, 1830 inclui duas espécies de maior tamanho (Catzeflis, 2018). Dentre eles, Rattus foi o único gênero registrado na Toca dos Ossos. O material inclui os dois maiores dentários presentes nas amostras. O comprimento da 

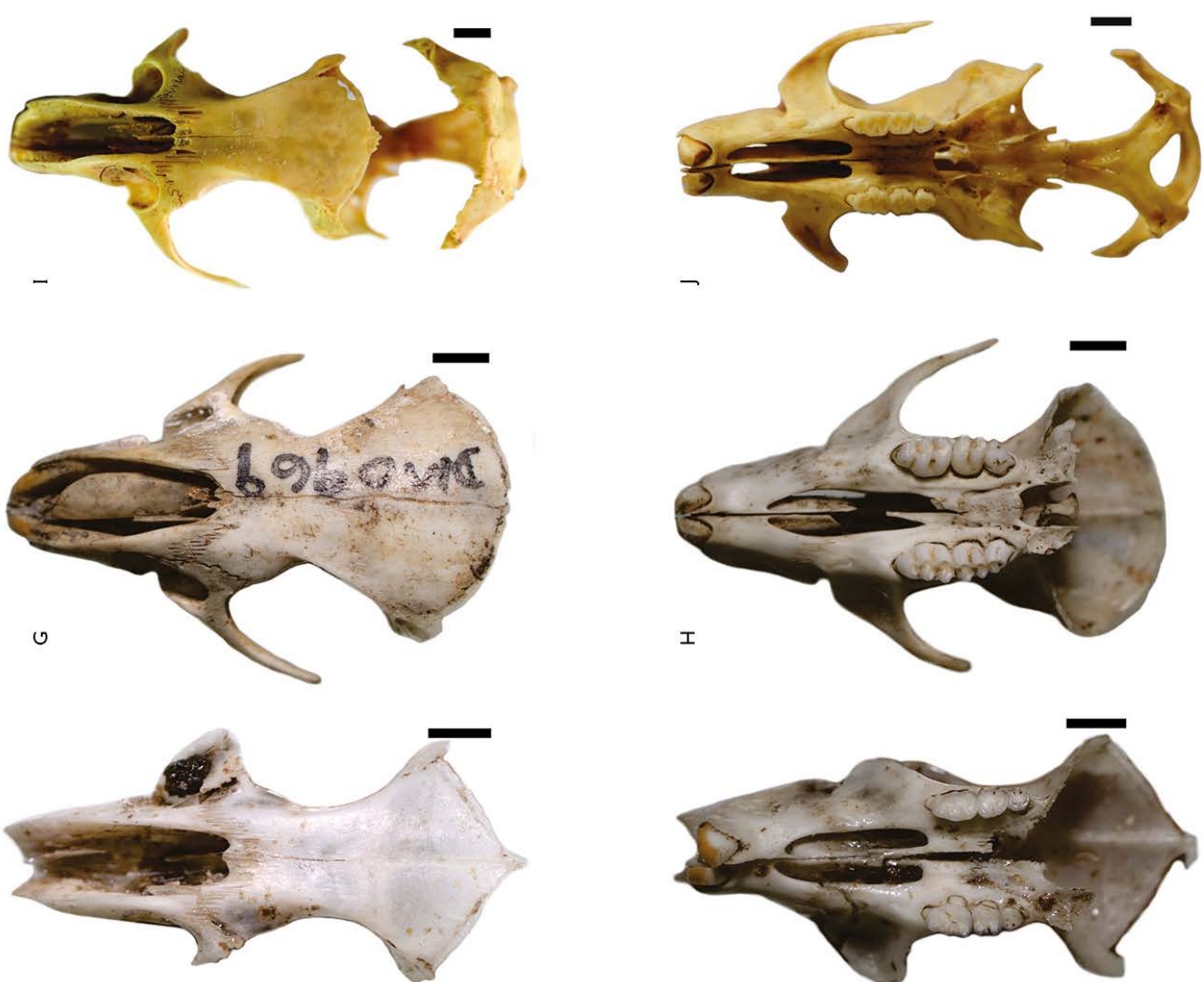

ш
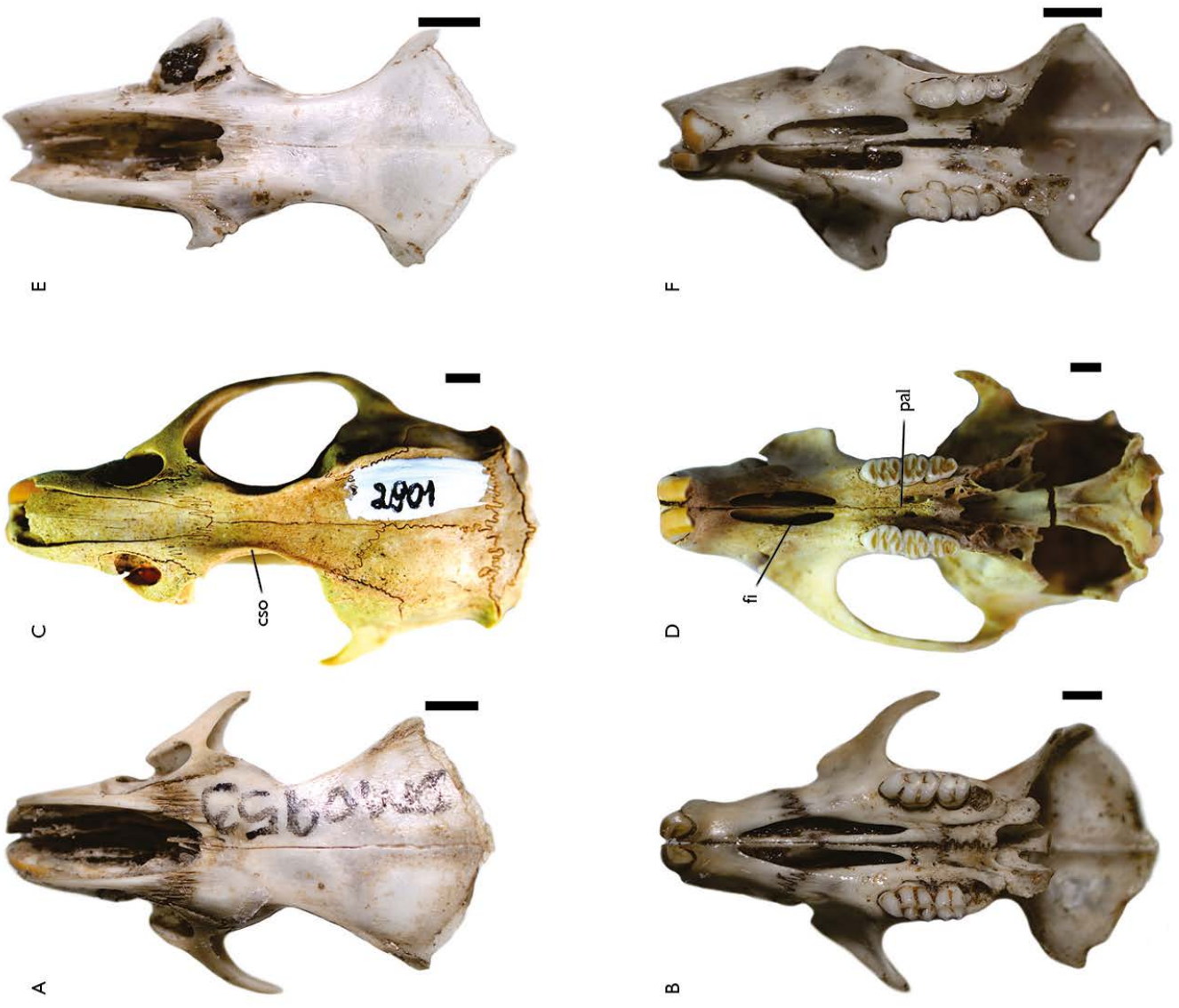

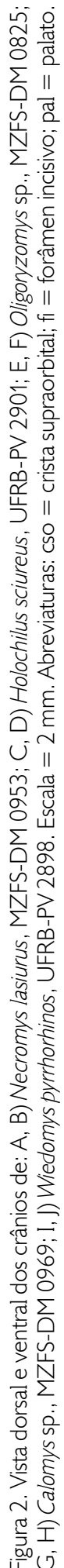


série molar inferior (Cm1-m3 $=5,94 \mathrm{~mm}$, MZFS-DM 0924) e o comprimento do $\mathrm{m} 1(\mathrm{Cm} 1=2,43 \mathrm{~mm}$, MZFS-DM 0839; Cm1 = 2,63 mm, MZFS-DM 0924) desses espécimes estão dentro da variação registrada para
R. $\operatorname{rattus}(\mathrm{Cm} 1-\mathrm{m} 3=5,8-6,7 \mathrm{~mm} ; \mathrm{Cm} 1=2,5-2,9 \mathrm{~mm})$ e abaixo dos valores para $R$. norvegicus (Berkenhout, 1769) (Cm1-m3 = 6,7-7,5 mm; Cm1 = 3,0-3,2 mm) (Catzeflis, 2018).

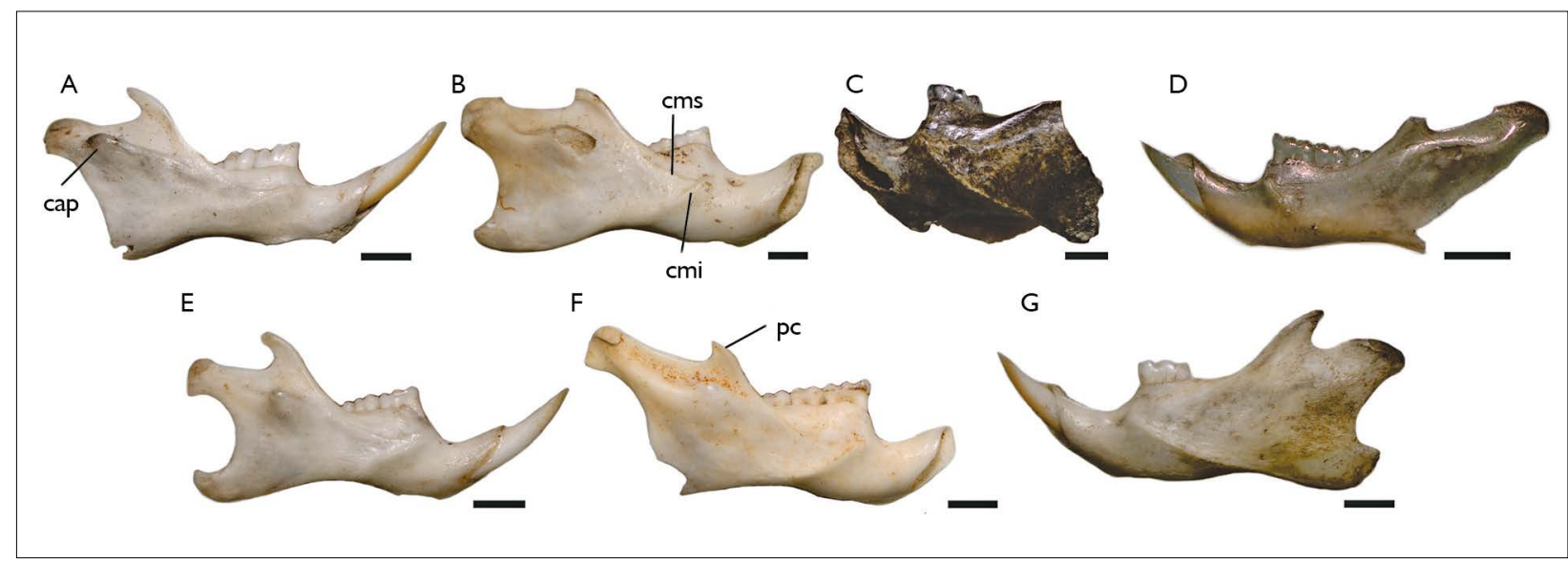

Figura 3. Vista lateral do dentário de: A) Necromys lasiurus, MZFS-DM 0831, direito; B) Cerradomys sp., MZFS-DM 0826, direito; C) Holochilus sciureus, UFRB-PV 908, esquerdo; D) Oligoryzomys sp., MZFS-DM 0867, esquerdo; E) Calomys sp., MZFS-DM 0835, direito; F) Wiedomys pyrrhorhinos, MZFS-DM 0838, direito; G) Rattus rattus, MZFS-DM 0839, esquerdo. Escala = 2 mm. Abreviaturas: cap = projeção capsular; $\mathrm{cmi}=$ crista massetérica inferior; $\mathrm{cms}=$ crista massetérica superior; $\mathrm{pc}=$ processo coronoide.
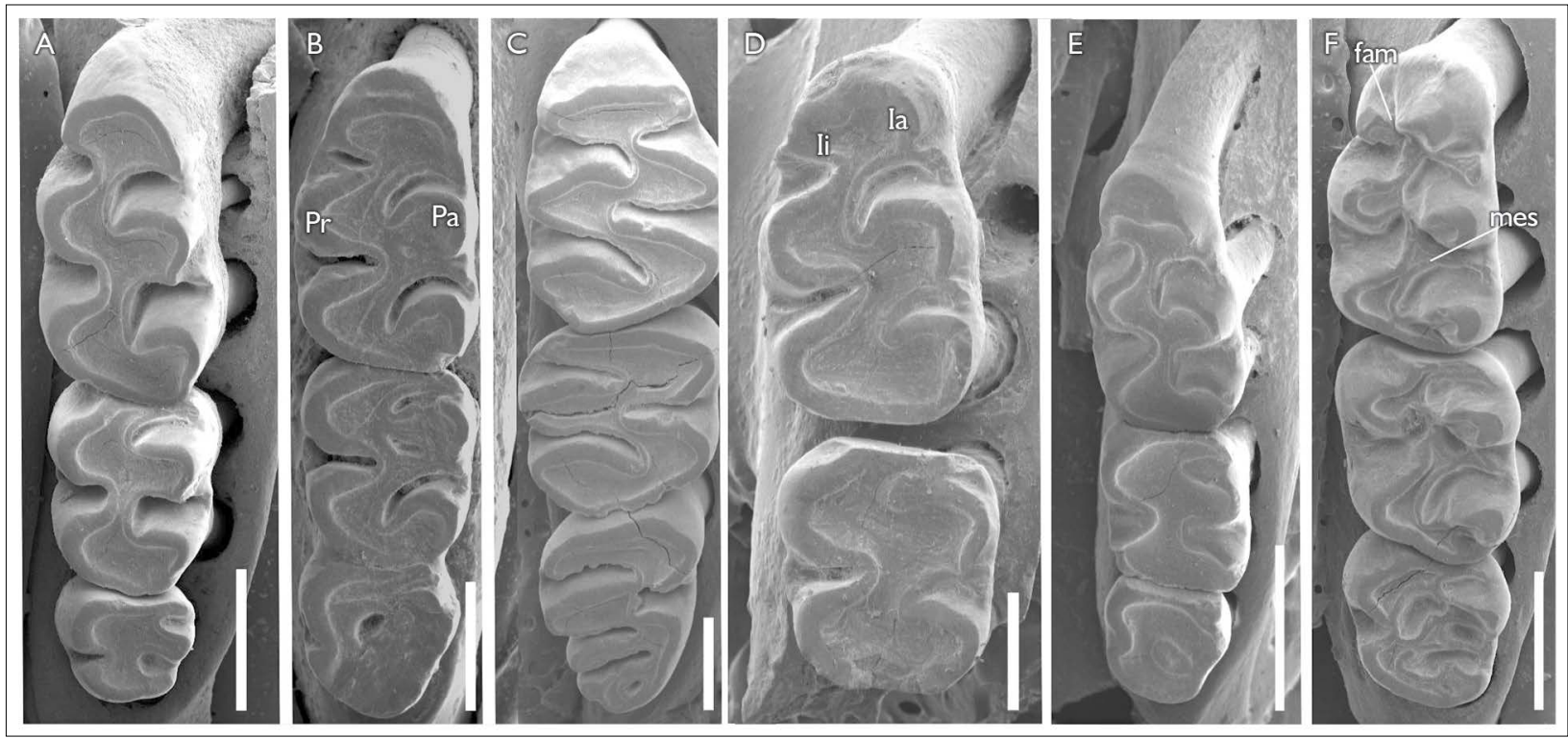

Figura 4. Vista oclusal dos molares superiores de: A) Necromys lasiurus, UFRB-PV 2748, M1-M3 esquerdos; B) Cerradomys sp., UFRB-PV 2861, M1-M3 esquerdos; C) Holochilus sciureus, UFRB-PV 2992, M1-M3 direitos; D) Oligoryzomys sp., UFRB-PV 2735, M1-M2 esquerdos; E) Calomys sp., UFRB-PV 2745, M1-M3 esquerdos; F) Wiedomys pyrrhorhinos, UFRB-PV 2823, M1-M3 esquerdos. Escala = 1 mm. Notar que o flexo anteromediano é indistinto devido ao desgaste dos molares em Oligoryzomys sp. e Calomys sp. Escala A, B, C, E, F = 1 mm; $\mathrm{D}=0,5 \mathrm{~mm}$. Abreviaturas: fam = flexo anteromediano; la = cônulo anterolabial; li = cônulo anterolingual; mes = mesolofo; Pa $=$ paracone; $\operatorname{Pr}=$ protocone.

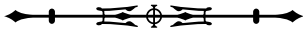




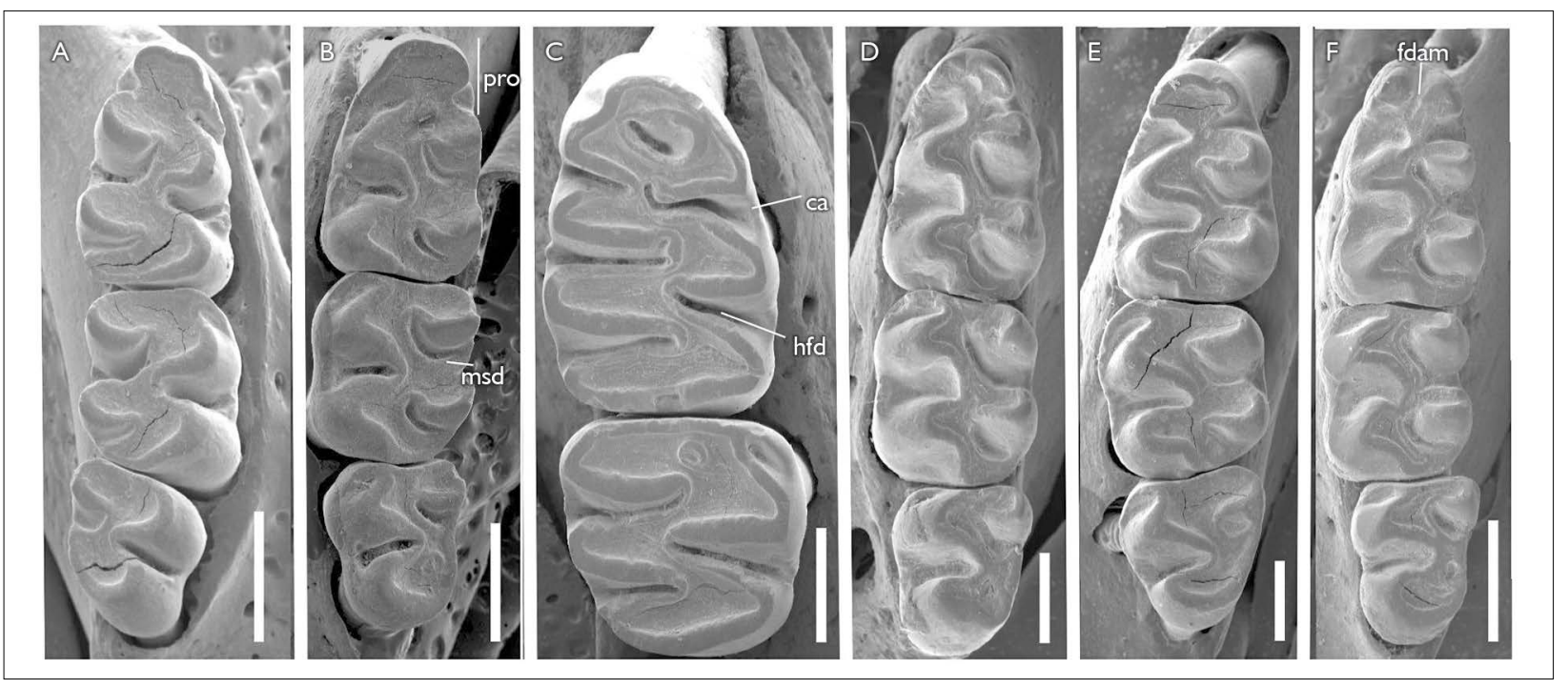

Figura 5. Vista oclusal dos molares inferiores de: A) Necromys lasiurus, MZFS-DM 0840, m1-m3 direitos; B) Cerradomys sp., MZFS-DM 0828, m1-m3 esquerdos; C) Holochilus sciureus, UFRB-PV 907, m1-m2 direitos; D) Oligoryzomys sp., MZFS-DM 0867, m1-m3 esquerdos; E) Calomys sp., MZFS-DM 0833, m1-m3 esquerdos; F) Wiedomys pyrrhorhinos, MZFS-DM 0836, m1-m3 esquerdos. Escala = 1 mm. Notar que o flexídeo anteromediano está pouco marcado devido ao desgaste dos molares em Oligoryzomys sp. e Calomys sp. Escala A, $\mathrm{B}, \mathrm{C}, \mathrm{F}=1 \mathrm{~mm} ; \mathrm{D}, \mathrm{E}=0,5 \mathrm{~mm}$. Abreviaturas: $\mathrm{ca}=$ cíngulo anterolabial; fdam = flexídeo anteromediano; $\mathrm{hfd}=$ hipoflexídeo; $\mathrm{msd}=$ mesolofídeo; pro = procíngulo.

\section{DISCUSSÃO}

Todos os táxons registrados neste trabalho possuem ocorrência atual na Caatinga e nas florestas estacionais associadas a este bioma, sendo Wiedomys pyrrhorhinos o único endêmico da Caatinga (Carmignotto \& Astúa, 2017). A riqueza registrada na Toca dos Ossos (sete espécies) representou 26,9\% dos roedores muroideos conhecidos para a Caatinga, que abriga 23 espécies de cricetídeos e três espécies de murídeos (Moojen, 1952; Carmignotto \& Astúa, 2017). Em comparação com outros levantamentos conduzidos nesse bioma, a maior parte dos estudos cuja amostragem se baseou na captura com armadilhas registrou riquezas menores, como no Parque Nacional de Ubajara, no Ceará (Guedes et al., 2000; três espécies); no Morro do Chapéu, Chapada Diamantina (Oliveira \& Pessôa, 2005; quatro espécies); em cinco localidades dos estados da Bahia e de Sergipe (Bezerra et al., 2014; quatro espécies); em Ouricuri, Pernambuco (Delciellos, 2016; quatro espécies); e no Parque Nacional do Catimbau, município de Buíque, Pernambuco
(Geise et al., 2010; cinco espécies). Por outro lado, Souza, M. et al. (2004) registraram 12 espécies de muroideos nos brejos de altitude nos estados da Paraíba e de Pernambuco. Assim, a análise dos egagropilos de corujas, dos quais provêm pelo menos parte do material da Toca dos Ossos (TO-I), demonstra ser uma importante ferramenta de amostragem de pequenos mamíferos, como já apontado em estudos prévios (e.g., Massoia, 1984; Bonvicino \& Bezerra, 2003; Formoso et al., 2016; Heisler et al., 2016).

A riqueza de roedores muroideos na Toca dos Ossos (sete espécies) foi intermediária àquelas obtidas em estudos com pelotas de Ty to furcata conduzidos em outros biomas. Por exemplo, três espécies foram registradas em uma área urbana e quatro em uma área rural na Mata Atlântica do estado de Pernambuco (Souza, D. et al., 2010); sete espécies em duas localidades no ecótono entre Amazônia e Cerrado do médio Araguaia (Rocha et al., 2011); oito espécies em uma restinga do estado do Rio de Janeiro (Lemos et al., 2015); dez espécies no Cerrado do estado da Bahia (Bonvicino \& Bezerra, 2003); e 13 espécies em

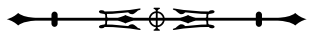


duas localidades em Floresta Ombrófila Mista na Mata Atlântica do estado de Santa Catarina (Cherem et al., 2018).

A diversidade encontrada nos egagropilos de coruja da TO-I foi semelhante à de outras cavernas estudadas na região Nordeste. Mancini et al. (2018) conduziram um levantamento de mamíferos recentes de pequeno porte através da análise de egagropilos de corujas em sete cavernas nos estados da Bahia e Sergipe. Esses autores obtiveram sete espécies de cricetídeos e duas de murídeos, e uma riqueza total variando de duas a nove espécies entre as cavernas, o que corresponde a uma espécie a mais para cada família, em comparação com os dados obtidos na Toca dos Ossos. Além disso, Mancini et al. (2018) registraram Calomys cf. expulsus como o táxon mais frequente entre os roedores muroideos, seguido por Necromys lasiurus, enquanto, na Toca dos Ossos, N. lasiurus foi a espécie mais abundante na TO-I e Calomys sp., a mais abundante na TO-II. Holochilus sciureus foi o único táxon aqui registrado que não foi encontrado na amostra analisada por Mancini et al. (2018).

Dados sobre roedores muroideos do Quaternário do Nordeste do Brasil são muito escassos. Castro et al. (2014) estudaram a assembleia de vertebrados da Gruta do Ioiô, no município de Palmeiras, Bahia, e reportaram apenas uma espécie de Sigmodontinae, Holochilus sciureus. Neves et al. (2017) analisaram a assembleia de sigmodontíneos da Serra da Capivara, estado do Piauí, e registraram sete táxons. Entre estes, Pseudoryzomys simplex (Winge, 1887) e Bibimys sp., gênero atualmente ausente na região Nordeste (Pardiñas et al., 2015a), não estiveram presentes na TO-II. O único táxon registrado em TO-II e que não estava entre os espécimes analisados por Neves et al. (2017) foi Oligoryzomys sp. Na amostra da Serra da Capivara, Calomys sp. foi o táxon mais frequente.

A amostra de TO-I representa material recente, visto que a espécie exótica Rattus rattus foi encontrada nos egagropilos de Ty to furcata. Por outro lado, o material proveniente da TO-II foi encontrado no sedimento de superfície da caverna e pode representar um intervalo de tempo maior. Auler et al. (2003) demonstraram que há considerável variação no tipo e na intensidade dos processos de acúmulo de material nas cavernas brasileiras, tanto na escala de regiões cársticas quanto de condutos dentro de uma mesma caverna, resultando em sítios fossilíferos complexos. Auler et al. (2006) analisaram a tafonomia relacionada a 19 espécies de megafauna da Toca dos Ossos e concluíram que esses fósseis foram transportados para o interior da caverna pelo escoamento de corpos d'água durante um ou vários momentos de maior precipitação. Os autores também demonstraram, através de datação de espeleotemas, que o período de deposição de fósseis nas cavernas brasileiras é bastante amplo, variando do Pleistoceno Médio ao Holoceno. Entretanto, não foi possível obter datas para a Toca dos Ossos no referido trabalho. Considerando a ausência de datações e de análise tafonômica detalhada do material de pequeno porte, não é possível indicar a idade do material da TO-II. No entanto, a ocorrência dos mesmos táxons na TO-I e na TO-II e sua similaridade com o registro atual na Caatinga (Carmignotto \& Astúa, 2017) podem sugerir que o material analisado proveniente da TO-II seja recente, tendo sido depositado após o estabelecimento local da Caatinga.

Os dados paleopalinológicos para a região noroeste da Bahia, onde se insere a Toca dos Ossos, indicam clima mais frio e úmido no início do Holoceno e a ocorrência de florestas úmidas de galeria, sendo que o estabelecimento das condições áridas teria ocorrido nos últimos 4.000 anos, levando a uma expansão da Caatinga (De Oliveira et al., 1997). A partir do registro de Myocastor coypus (Molina, 1782), Cartelle \& Lessa (1989) sugeriram clima mais úmido e frio durante o fim do Pleistoceno e início do Holoceno, com vegetação provavelmente composta por matas ciliares e cerrado. Lessa et al. (1998) também registraram espécies de hábitos florestais - e.g., Eira barbara (Linnaeus, 1758) - e semiaquáticos - e.g., Lontra longicaudis (Olfers, 1818) - entre os carnívoros fósseis coletados na Toca dos Ossos. 
A análise e a identificação de fragmentos pertencentes a outros grupos de pequenos mamíferos coletados na Toca dos Ossos, como marsupiais e roedores caviomorfos, constituem importante etapa subsequente do estudo dessa caverna do Nordeste brasileiro. Além disso, a análise tafonômica do material de pequenos mamíferos da Toca dos Ossos também deverá ser objeto dos próximos estudos e poderá auxiliar no entendimento da assembleia dessa caverna. Esses dados serão importantes para a compreensão dos pequenos mamíferos em uma região ainda pouco amostrada, como a Caatinga.

\section{CONCLUSÃO}

A análise de 210 fragmentos cranianos e mandibulares de roedores muroideos provenientes da Toca dos Ossos, no bioma Caatinga, resultou no registro de 99 indivíduos, pertencentes a duas famílias, Cricetidae e Muridae, e sete espécies (Necromys lasiurus, Cerradomys sp., Holochilus sciureus, Oligoryzomys sp., Calomys sp., Wiedomys pyrrhorhinos e Rattus rattus). Essa riqueza é considerável e relevante, comparada a outros trabalhos sobre a mastofauna deste bioma. Apesar de muito semelhantes taxonomicamente, as amostras dos dois pontos de coleta (TO-I e TO-II) apresentaram diferenças nas frequências dos táxons. No entanto, ambas as áreas da Toca dos Ossos incluíram somente táxons de ocorrência atual na Caatinga, o que sugere que o material de TO-II também é bastante recente, tendo sido depositado após o estabelecimento deste bioma na região, o qual ocorreu há cerca de 4.000 anos.

\section{AGRADECIMENTOS}

Agradecemos à Universidade Federal do Recôncavo da Bahia (UFRB), pela infraestrutura concedida; ao Laboratório Central de Microscopia Eletrônica da UFSC, pelas fotografias em MEV. Ao Sr. Admir Brunelli, pelo apoio aos trabalhos de campo. À editora Alexandra Bezerra e a três revisores anônimos, por suas correções e sugestões nas versões preliminares do texto. Ao Conselho Nacional de
Desenvolvimento Científico e Tecnológico (CNPq), através dos editais MCT/CNPq 14/2010 - Universal (475291/20107) e MCT/CNPq 14/2011 - Universal (475287/2011-8).

\section{REFERÊNCIAS}

ALVARES, C. A., J. L. STAPE, P. C. SENTELHAS, G. MORAES, J. LEONARDO \& G. SPAROVEK, 2013. Köppen's climate classification map for Brazil. Meteorologische Zeitschrift 22(6): 711-728. DOI: https://doi.org/10.1127/0941-2948/2013/0507

ANDERSON, S. \& N. OLDS, 1989. Notes on Bolivian mammals. 5. Taxonomy and distribution of Bolomys (Muridae, Rodentia). American Museum Novitates (2935): 1-22.

AULER, A. S., L. B. PILÓ, P. L. SMART, X. WANG, W. A. NEVES \& R. L. EDWARDS, 2003. Cronologia e processos de deposição da megafauna quaternária em cavernas brasileiras. Anais do Congresso sobre Planejamento e Gestão das Zonas Costeiras dos Países de Expressão Portuguesa/Congresso da Associação Brasileira de Estudos do Quaternário/Congresso do Quaternário dos Países de Língua Ibérica 2/9/2: 1 CD-ROM.

AULER, A. S., L. B. PILÓ, P. L. SMART, X. WANG, D. HOFFMANN, D. A. RICHARDS, R. L. EDWARDS, W. A. NEVES \& H. CHENG, 2006. U-series dating and taphonomy of Quaternary vertebrates from Brazilian caves. Palaeogeography, Palaeoclimatology, Palaeoecology 240: 508-522.

BARBIÈRE, F., P. E. ORTIZ \& U. F. J. PARDIÑAS, 2019. The oldest sigmodontine rodent revisited and the age of the first South American cricetids. Journal of Paleontology 93(2): 368-384. DOI: https://doi. org/10.1017/jpa.2018.74

BEISAW, A. M., 2013. Identifying and interpreting animal bones: a manual: 1-214. Texas A \& M University Press, College Station.

BEZERRA, A. M. R., A. LAZAR, C. R. BONVICINO \&J. MARINHO FILHO, 2013. Wiedomys cerradensis Gonçalves, Almeida and Bonvicino, 2005 (Mammalia: Rodentia: Cricetidae) in Tocantins and Goiás states, central- northern Brazil. Check List 9(3): 680-83. DOI: https://doi.org/10.15560/9.3.680

BEZERRA, AM. R., A. LAZAR, C. R. BONVICINO \&A. S. CUNHA, 2014. Subsidies for a poorly known endemic semiarid biome of Brazil: non-volant mammals of an eastern region of Caatinga. Zoological Studies 53: 16. DOI: https://doi.org/10.1186/1810-522X-53-16

BONVICINO, C. R., 2015. Tribe Wiedomyini Reig, 1980. In: J. L. PATTON, U. F. J. PARDIÑAS \& G. D'ELÍA (Ed.): Mammals of South America: vol. 2: Rodents: 682-685. University of Chicago Press, Chicago.

BONVICINO, C. R. \& A. M. R. BEZERRA, 2003. Use of regurgitated pellets of Barn Owl (Tyto alba) for inventorying small mammals in the cerrado of Central Brazil. Studies on Neotropical Fauna and Environment 38(1): 1-5. DOI: http://doi.org/10.1076/ snfe.38.1.1.14030 
BONVICINO, C. R., F. CASADO \& M. WEKSLER, 2014. A new species of Cerradomys (Mammalia: Rodentia: Cricetidae) from Central Brazil, with remarks on the taxonomy of the genus. Zoologia 31(6): 525-540. DOI: http://dx.doi.org/10.1590/S198446702014000600002

BRANDÃO, M. V. \& F. O. NASCIMENTO, 2015. On the occurrence of Holochilus chacarius (Cricetidae: Sigmodontinae) in Brazil, with taxonomic notes on Holochilus species. Papéis Avulsos de Zoologia 55(3): 47-67. DOI: http://dx.doi.org/10.1590/0031-1049.2015.55.03

BURGIN, C. J., J. P. COLELLA, P. L. KAHN \& N. S. UPHAM, 2018. How many species of mammals are there? Journal of Mammalogy 99(1): 1-14. DOI: https://10.1093/Jmammal/gyx147

CACCAVO, A. \& J. A. OLIVEIRA, 2016. Detecting morphological limits between parapatric species: cranial variation in Cerradomys (Cricetidae: Sigmodontinae) from northeastern Brazil. Journal of Mammalogy 97(6): 1602-1616. DOI: https://doi.org/10.1093/ jmammal/gyw124

CARMIGNOTTO, A. P. \& D. ASTÚA, 2017. Mammals of the Caatinga: diversity, ecology, biogeography, and conservation. In: J. M. C. SILVA, I. R. LEAL \& M. TABARELLI (Ed.): Caatinga: 211-254. Springer, Cham.

CARTELLE, C. \& G. LESSA, 1988. Descrição de um novo gênero e espécie de Macraucheniidae (Mammalia, Litopterna) do Pleistoceno do Brasil. Paulacoutiana 3: 3-26.

CARTELLE, C. \& G. LESSA, 1989. Presença de Myocastor coypus (Molina, 1782), Rodentia, Myocastoridae, do Pleistoceno Final e Holoceno, no centro-oeste da Bahia. Anais do Congresso Brasileiro de Paleontologia 11: 583-591.

CARTELLE, C. \& V. S. ABUHID, 1994. Chiroptera do Pleistoceno final-Holoceno da Bahia. Acta Geológica Leopoldensia 39: 429-440.

CARVALHO, M. S. \& T. V. OLIVEIRA, 2015. Small non-volant mammals (Didelphimorphia and Rodentia) from the RPPN Guarirú, an Atlantic Forest fragment in northeastern Brazil. Check List 11(6): 1-9. DOI: http://dx.doi.org/10.15560/11.6.1782

CASTRO, M. C., F. C. MONTEFELTRO \& M. C. LANGER, 2014 The Quaternary vertebrate fauna of the limestone cave Gruta do Ioiô, northeastern Brazil. Quaternary International 352: 164-175. DOI: http://dx.doi.org/10.1016/j.quaint.2014.06.038

CATZEFLIS, F., 2018. Invasive rodents in French Guiana: an overview for Mus musculus, Rattus norvegicus, and R. rattus (Murinae: Muridae). Boletim da Sociedade Brasileira de Mastozoologia 81: 1-9.

CHEREM, J. J., P. HADLER, N. S. STUTZ \& U. F. J. PARDIÑAS, 2018. Pequenos mamíferos (Didelphimorphia, Chiroptera e Rodentia) em egagropilos de Tyto furcata (coruja-das-igrejas) (Aves, Tytonidae) do sul do Brasil. Biotemas 31(3): 43-58. DOI: http:// dx.doi.org/10.5007/2175-7925.2018v31n3p43
COMITÊ DA BACIA HIDROGRÁFICA DO RIO SALITRE (CBHS), 2017. Plano de recursos hídricos e proposta de enquadramento dos corpos de água da Bacia Hidrográfica do Rio Salitre: síntese executiva: 1-242. Instituto do Meio Ambiente e Recursos Hídricos, Salvador.

CZAPLEWSKI, N. J. \& C. CARTELLE, 1998. Pleistocene bats from cave deposits in Bahia, Brazil. Journal of Mammalogy 79(3): 784-803.

DELCIELLOS, A. C., 2016. Mammals of four Caatinga areas in northeastern Brazil: inventory, species biology, and community structure. Check List 12(3): 1-15. DOI: http://dx.doi. org/10.15560/12.3.1916

DE OLIVEIRA, P. E., A. M. F. BARRETO \& K. SUGUIO, 1997. Paleovegetação e paleoclima do Quaternário tardio da caatinga brasileira: o campo de dunas do médio rio São Francisco, Bahia. Resumos Expandidos do Congresso da Associação Brasileira de Estudos do Quaternário 6: 171-175.

FABRE, P. H., L. HAUTIER, D. DIMITROV \& E. DOUZERY, 2012. A glimpse on the pattern of rodent diversification: a phylogenetical approach. BMC Evolutionary Biology 12: 88. DOI: https://doi. org/10.1186/1471-2148-12-88

FLYNN, L. J., L. L. JACOBS \& E. H. LINDSAY, 1985. Problems in muroid phylogeny: relationship to other rodents and origin of major groups. In: W. P. LUCKETT \& J. L. HARTENBERGER (Ed.): Evolutionary relationships among rodents: 589-616. Springer, Boston. DOI: https://doi.org/10.1007/978-1-4899-0539-0_22

FORMOSO, A. E., P. TETA, A. E. CARBAJO \& U. F. J. PARDIÑAS, 2016. Unraveling the patterns of small mammal species richness in the southernmost aridlands of South America. Journal of Arid Environments 134: 136-144. DOI: https://doi.org/10.1016/j. jaridenv.2016.07.007

FREITAS, R. R., P. L. B. ROCHA \& P. C. SIMÕES-LOPES, 2005. Habitat structure and small mammals abundances in one semiarid landscape in the Brazilian Caatinga. Revista Brasileira de Zoologia 22(1): 119-129. DOI: http://dx.doi.org/10.1590/S010181752005000100015

GEISE, L., 2012. Akodon cursor. Mammalian Species 44(893): 33-43.

GEISE, L. \& L. G. PEREIRA, 2008. Rodents (Rodentia) and marsupials (Didelphimorphia) in the municipalities of Ilhéus and Pau Brasil, state of Bahia, Brazil. Check List 4(2): 174-177. DOI: http://dx.doi. org/10.15560/4.2.174

GEISE, L., R. PARESQUE, H. SEBASTIÃO, L. T. SHIRAI, D. ASTÚA \& G. MARROIG, 2010. Non-volant mammals, Parque Nacional do Catimbau, Vale do Catimbau, Buíque, state of Pernambuco, Brazil, with karyologic data. Check List 6(1): 180-186. DOI: http://dx.doi. org/10.15560/6.1.180

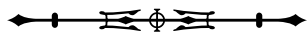


GOMES, A. C. F., G. LESSA, C. CARTELLE \& L. KERBER, 2019. New fossil remains of Quaternary capybaras (Rodentia: Caviomorpha: Caviidae) from the intertropical region of Brazil: morphology and taxonomy. Journal of South American Earth Sciences 91: 36-46. DOI: https://doi.org/10.1016/j.jsames.2019.01.006

GUEDES, P. G., S. S. P. SILVA, A. R. CAMARDELLA, M. F. G. ABREU, D. M. BORGES-NOJOSA, J. A. G. SILVA \& A. A. SILVA, 2000. Diversidade de mamíferos do Parque Nacional de Ubajara (Ceará, Brasil). Mastozoología Neotropical 7(2): 95-100.

HEISLER, L. M., C. M. SOMERS \& R. G. POULIN, 2016. Owl pellets: a more effective alternative to conventional trapping for broad-scale studies of small mammal communities. Methods in Ecology and Evolution 7(1): 96-103. DOI: https://doi.org/10.1111/2041-210X.12454

HONEYCUTT, R. L., L. J. FRABOTTA\& D. L. ROWE, 2007. Rodent evolution, phylogenetics and biogeography. In: J. O. WOLFF \& P. W. SHERMAN (Ed.): Rodent societies: an ecological \& evolutionary perspective: 8-23. The University of Chicago Press, Chicago.

INSTITUTO DO MEIO AMBIENTE E RECURSOS HÍDRICOS (INEMA), 2016. Portaria $n^{\circ}$ 13.203, de 27 de dezembro de 2016. Diário Oficial do Estado da Bahia 22084: 44-45.

JANSA, S. A. \& M. WEKSLER, 2004. Phylogeny of muroid rodents: relationships within and among major lineages as determined by IRBP gene sequences. Molecular Phylogenetics and Evolution 31(1): $256-$ 276. DOI: https://doi.org/10.1016/j.ympev.2003.07.002

JESUS, D. S. \& T. V. OLIVEIRA, 2017. Morcegos (Mamalia, Chiroptera) em egagrópilas da coruja-das-torres (Tyto furcata) em uma caverna do Nordeste brasileiro. Boletim do Museu de Biologia Mello Leitão, Nova Série 39(1): 69-93.

LEAL, I. R., J. M. C. SILVA, M. TABARELLI \& T. E. LACHER, 2005. Mudando o curso da conservação da biodiversidade na Caatinga do Nordeste do Brasil. Megadiversidade 1(1): 139-146.

LEMOS, H. M., C. A. O. SILVA, F. M. PATIU \& P. R. GONÇALVES, 2015. Barn Owl pellets (Aves: Tyto furcata) reveal a higher mammalian richness in the Restinga de Jurubatiba National Park, Southeastern Brazil. Biota Neotropica 15(2): e20140121. DOI: http://dx.doi. org/10.1590/1676-06032015012114

LESSA, G., C. CARTELLE, H. D. FARIA \& P. R. GONÇALVES, 1998. Novos achados de mamíferos carnívoros do Pleistoceno FinalHoloceno em grutas calcárias do estado da Bahia. Acta Geologica Leopoldensia 21(46-47): 157-169.

LESSA, G., P. R. GONÇALVES \& L. M. PESSÔA, 2005. Variação geográfica em caracteres cranianos quantitativos de Kerodon rupestris (Wied, 1820) (Rodentia, Caviidae). Arquivos do Museu Nacional 63(1): 75-88.

LESSA, G. \& L. M. PESSÔA, 2005. Variação ontogenética e sexual em caracteres cranianos de Kerodon rupestris Wied, 1820, (Rodentia: Caviidae). Arquivos do Museu Nacional 63(3): 599-618.
LOBO, L. S., G. LESSA, C. CARTELLE \& P. S. R. ROMANO, 2017. Dental eruption sequence and hypsodonty index of a Pleistocene macraucheniid from the Brazilian Intertropical Region. Journal of Paleontology 91(5): 1083-1090. DOI: https://doi.org/10.1017/ jpa.2017.54

MACHADO, L. F., R. PARESQUE \& A. U. CHRISTOFF, 2011. Anatomia comparada e morfometria de Oligoryzomys nigripes e O. flavescens (Rodentia, Sigmodontinae) no Rio Grande do Sul, Brasil. Papéis Avulsos de Zoologia 51(3): 29-47. DOI: http:// dx.doi.org/10.1590/S0031-10492011000300001

MANCINI, M. C. S., P. R. O. ROTH, P. G. G. BRENNAND, J. M. R. E. AGUILAR \& P. A. ROCHA, 2018. Tyto furcata (Tytonidae: Strigiformes) pellets: tools to access the richness of small mammals of a poorly known Caatinga area in northeast Brazil. Mammalia 83(4): 390-398. DOI: http://dx.doi.org/10.1515/ mammalia-2018-0017

MASSOIA, E., 1984. La importancia de los análisis de regurgitados de aves estrigiformes. ACINTACNIA, Asociación Cooperadora INTA 1(11): 38-39.

MOOJEN, J., 1952. Os roedores do Brasil: 1-214. Instituto Nacional do Livro, Rio de Janeiro.

NEVES, S. B., E. L. MAYER, P. HADLER \& A. M. RIBEIRO, 2017. Sigmodontine rodents (Rodentia, Cricetidae) from Quaternary cave deposits of Serra da Capivara, northeastern Brazil. Revista Brasileira de Paleontologia 20(2): 163-178. DOI: http://dx.doi. org/10.4072/rbp.2017.2.02

OLIVEIRA, J. A., P. R. GONÇALVES \& C. R. BONVICINO, 2003. Mamíferos da Caatinga. In: J. A. LEAL, M. TABARELLI \& J. M. C. SILVA (Ed.): Ecologia e conservação da Caatinga: 275-334. Editora Universitária da UFPE, Recife.

OLIVEIRA, J. A. \& L. M. PESSÔA, 2005. Mamíferos. In: F. A. JUNCÁ, L. FUNCH \& W. ROCHA (Ed.): Biodiversidade e conservação da Chapada Diamantina: 377-405. Ministério do Meio Ambiente, Brasília.

PARDIÑAS, U. F. J. \& P. TETA, 2011. Fossil history of the marsh rats of the genus Holochilus and Lundomys (Cricetidae, Sigmodontinae) in southern South America. Estudios Geológicos 67(1): 111-129. DOI: https://doi.org/10.3989/egeol.40347.136

PARDIÑAS, U. F. J., G. D'ELÍA \& P. TETA. 2015a. Genus Bibimys Massoia, 1979. In: J. L. PATTON, U. F. J. PARDIÑAS \& G. D'ELÍA (Ed.): Mammals of South America: vol. 2: Rodents: 204-208. University of Chicago Press, Chicago.

PARDIÑAS, U. F. J., P. TETA, P. E. ORTIZ, J. P. JAYAT \& J. SALAZARBRAVO, 2015b. Genus Necromys Ameghino, 1889. In: J. L. PATTON, U. F. J. PARDIÑAS \& G. D'ELIA (Ed.): Mammals of South America: vol. 2: Rodents: 232-247. University of Chicago Press, Chicago.

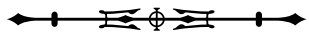


PARDIÑAS, U. F. J., P. MYERS, L. LEÓN-PANIAGUA, N. O. GARZA, J. COOK, B. KRYŠTUFEK, R. HASLAUER, R. BRADLEY, G. SHENBROT \& J. L. PATTON, 2017. Family Cricetidae. In: D. E. WILSON, T. E. LACHER \& R. A. MITTERMEIER (Ed.): Handbook of the mammals of the world: vol. 7: Rodents II: 204-279. Lynx Ediciones, Barcelona.

PATTON, J., U. F. J. PARDIÑAS \& G. D'ELÍA, 2015. Mammals of South America: vol. 2: Rodents: 1-1336. The University of Chicago Press, Chicago.

PERCEQUILLO, A. R., 2006. Guia para a nomenclatura e padronização da descrição da dentição nos roedores sigmodontíneos. Boletim da Sociedade Brasileira de Mastozoologia (47): 5-11.

PERCEQUILLO, A. R., E. HINGST-ZAHER \& C. R. BONVICINO, 2008. Systematic review of genus Cerradomys Weksler, Percequillo and Voss, 2006 (Rodentia: Cricetidae: Sigmodontinae: Oryzomyini), with description of two new species from eastern Brazil. American Museum Novitates (3622): 1-46. DOI: https://doi.org/10.1206/495.1

PEREIRA, L. G. \& L. GEISE, 2009. Non-flying mammals of Chapada Diamantina (Bahia, Brazil). Biota Neotropica 9(3): 185-196. DOI: http://dx.doi.org/10.1590/S1676-06032009000300019

REIG, O. A., 1977. A proposed unified nomenclature for the enamelled components of the molar teeth of the Cricetidae (Rodentia). Journal of Zoology 181(2): 227-241. DOI: http://doi. org/10.1111/j.1469-7998.1977.tb03238.x

RIZZINI, C. T., 1997. Tratado de fitogeografia do Brasil: 1-747. Âmbito Cultural Edições Ltda., Rio de Janeiro.

ROCHA, R. G., E. FERREIRA, Y. L. LEITE, C. FONSECA \& L. P. COSTA, 2011. Small mammals in the diet of barn owls, Tyto alba (Aves: Strigiformes) along the mid-Araguaia river in central Brazil. Zoologia 28(6): 709-716. DOI: https://doi.org/10.1590/S198446702011000600003

RUBBIOLI, E., A. AULER, D. MENIN \& R. BRANDI, 2019. Cavernas - Atlas do Brasil subterrâneo: 1-340. Instituto Chico Mendes de Conservação da Biodiversidade, Brasília.

SALAZAR-BRAVO, J., 2015. Genus Calomys Waterhouse, 1837. In: J. L. PATTON, U. F. J. PARDIÑAS \& G. D'ELÍA (Ed.): Mammals of South America: vol. 2: Rodents: 481-507. University of Chicago Press, Chicago.

SBRAGIA, I. A. \&A. CARDOSO, 2008. Quiropterofauna (Mammalia: Chiroptera) cavernícola da Chapada Diamantina, Bahia, Brasil. Chiroptera Neotropical 14(1): 360-365.
SILVA, J. M. C., I. R. LEAL \& M. TABARELLI, 2017. Preface. In: J. M. C. SILVA, I. R. LEAL \& M. TABARELLI (Ed.): Caatinga: vii-x. Springer, Cham.

SOUZA, M. A. N., A. LANGGUTH \& E. A. GIMENEZ, 2004. Mamíferos dos brejos de altitude em Paraíba e Pernambuco. In: K. C. PORTO, J. J. P. CABRAL \& M. TABARELLI (Org.): Brejos de altitude em Pernambuco e Paraíba: história natural, ecologia e conservação: 229-254. Ministério do Meio Ambiente, Brasília.

SOUZA, D. P., P. H. ASFORA, T. C. LIRA \& D. ASTÚA, 2010. Small mammals in Barn Owl (Tyto alba - Aves, Strigiformes) pellets from northeastern Brazil, with new records of Gracilinanus and Cryptonanus (Didelphimorphia, Didelphidae). Mammalian Biology 75(4): 370374. DOI: http://doi.org/10.1016/J.mambio.2009.08.003

STUTZ, N. S., P. HADLER, J. J. CHEREM, F. J. FERNÁNDEZ, U. F. J. PARDIÑAS \& A. M. RIBEIRO, 2018. Noteworthy sigmodontine (Rodentia: Cricetidae) diversity in southern Brazil as an indication of environmental change during the Holocene. Historical Biology 32(5): 649-670. DOI: https://doi.org/10.1080/08912963.2018.15 24470

VAZ, S. M., 2005. Mamíferos colecionados pelo serviço de estudos e pesquisas sobre a febre amarela nos municípios de llhéus e Buerarema, estado da Bahia, Brasil. Arquivos do Museu Nacional 63(1): 21-28.

WEKSLER, M. \& C. R. BONVICINO, 2005. Taxonomy of pigmy rice rats (genus Oligoryzomys, Rodentia: Sigmodontinae) of the Brazilian Cerrado, with the description of two new species. Arquivos do Museu Nacional 63(1): 113-130.

WEKSLER, M., A. R. PERCEQUILLO \& R. S. VOSS, 2006. Ten new genera of oryzomyine rodents. American Museum Novitates 3537: 1-29.

WEKSLER, M. \& C. R. BONVICINO, 2015. Genus Oligoryzomys Bangs, 1900. In: J. L. PATTON, U. F. J. PARDIÑAS \& G. D'ELIA(Ed.): Mammals of South America: vol. 2: Rodents: 417-437. University of Chicago Press, Chicago.

WEKSLER, M., E. M. S. LEMOS, P. S. D'ANDREA \& C. R. BONVICINO, 2017. The taxonomic status of Oligoryzomys mattogrossae (Allen 1916) (Rodentia: Cricetidae: Sigmodontinae), reservoir of Anajatuba Hantavirus. American Museum Novitates (3880): 1-32. DOI: https://doi.org/10.1206/3880.1

WILSON, D. E. \& D. M. REEDER, 2005. Mammal species of the world: a taxonomic and geographic reference: 3. ed.: 1-2142. The Johns Hopkins University Press, Baltimore. 
Apêndice 1. Lista dos espécimes depositados na Coleção de Mamíferos do Museu de Zoologia da Universidade Estadual de Feira de Santana (MZFS-DM) e do Departamento de Ecologia e Zoologia da Universidade Federal de Santa Catarina (UFSC) utilizados para a comparação com os fragmentos amostrados na Toca dos Ossos.

Akodon cursor - UFSC 4712, 4785.

Calomys tener - UFSC 5166, 5490.

Cerradomys subflavus - MZFS-DM 0202; UFSC 2248, 2251, 2254, 2257, 2258, 2260, 2280.

Cerradomys vivoi - MZFS-DM 0109, 0111; UFSC 5222, 5223.

Euryoryzomys russatus - UFSC 3940, 3944, 4891, 5689.

Holochilus grupo brasiliensis - UFSC 5074, 5890.

Holochilus sciureus - UFSC 2754, 2755.

Hylaeamys laticeps - MZFS-DM 0003, 0004, 0010, 0020.

Necromys lasiurus - MZFS-DM 0033; UFSC 3599, 3600, 3876, 4714, 4815, 5212.

Oecomys catherinae - UFSC 2277, 2278, 2279.

Oligoryzomys sp. - UFSC 2525, 2527, 2530, 3707, 3950, 3951, 4399, 4717, 4791, 4792, 6025.

Rhipidomys sp. - MZFS-DM 0651.

Rhipidomys mastacalis - MZFS-DM 0025.

Wiedomys cerradensis - UFSC 6191.

Wiedomys pyrrhorhinos - UFSC 2822, 2823, 2827, 2828, 2829, 5224. 
Apêndice 2. Lista do material estudado da TO-I depositado na Coleção de Mamíferos do Museu de Zoologia da Universidade Estadual de Feira de Santana (MZFS-DM) e da TO-II na Coleção de Paleovertebrados da Universidade Federal do Recôncavo da Bahia (UFRB-PV). Os dentes, se presentes, são indicados entre parênteses. Abreviaturas: Cr: crânio; dir = direito; Dn = dentário; esq = esquerdo; $M=$ molar superior; $\mathrm{m}=$ molar inferior; $\mathrm{Mx}=$ maxilar.

\section{TO-I}

Calomys sp.: Cr: MZFS-DM 0876, 0968 (M1-M3 dir e M1-M3 esq); 0964 (M2-M3 dir e M1-M3 esq); 0966 (M1-M3 dir e M1 esq); Dn dir: MZFS-DM 0835, 0846, 0877 (m1-m3); Dn esq: MZFS-DM 0829, 0833 (m1-m3). Cerradomys sp.: Mx dir: MZFS-DM 0815 (M1-M2); Dn dir: MZFS-DM 0826 (m1-m3); Dn esq: MZFS-DM 0828 (m1-m3). Holochilus sciureus: Cr: MZFS-DM 0950 (M1-M3 dir e M1-M3 esq); Mx dir: MZFS-DM 0913 (M1). Necromys lasiurus: Cr: MZFS-DM 0857, 0948, 0949, 0951, 0952, 0953, 0959, 0967, 0969 (M1-M3 dir e M1-M3 esq); 0957 (M1-M2 dir e M1-M3 esq); 0954, 0958, 0963 (M1-M2 dir e M1-M2 esq); 0983 (M1-M2 dir); 0960 (M1 e M3 dir e M1 esq); 0961 (M1 dir e M1 esq); Mx dir: MZFS-DM 0821, 0823, 0866 (M1-M2); 0824, 0864 (M1); Mx esq: MZFS-DM 0818, 0820, 0821, 0823, 0824 (M1-M3); 0816, 0865 (M1-M2); 0817 (M1); Dn dir: MZFS-DM 0840, 0843 (m1-m3); 0841, 0844 (m1-m2); 0842,0863 (m1); Dn esq: MZFS-DM 0847, 0848, 0852, 0858, 0859 (m1-m3); 0849, 0853, 0860 (m1-m2); 0831, 0834, 0837, 0845, 0854, 0855, 0861, 0862, 0874, 0875 (m1). Oligoryzomys sp.: Cr: MZFS-DM 0825 (M1-M3 dir e M1-M3 esq); Dn dir: MZFS-DM 0856 (m1); Dn esq: MZFS-DM 0867 (m1-m3). Wiedomys pyrrhorhinos: Cr: MZFS-DM 0691, 0905, 0946, 0956, 0965, 2907 (M1-M3 dir e M1-M3 esq); 0955 (M2-M3 dir e M1-M3 esq); Mx esq: MZFS-DM 0822 (M1-M2); 0819 (M1); Dn dir: MZFS-DM 0832 (m1-m3), 0850 (m1-m2); Dn esq: MZFS-DM 0836, 0838 (m1-m3). Rattus rattus: Dn esq: MZFS-DM 0924 (m1-m3); 0839 (m1).

\section{TO-II}

Calomys sp.: Cr: UFRB-PV 2862 (M1-M3 dir e M1-M3 esq); UFRB-PV 2960 (M1-M3 dir e M3 esq); Mx dir: UFRBPV 3132 (M1-M3); 2746 (M1-M2); 951 (M1); Mx esq: UFRB-PV 2738, 2745, 2863, 2955 (M1-M3); 2991, 3157 (M1-M2); Dn dir: UFRB-PV 2714; 2873, 2874, 2935, 2937, 2939, 2994, 3007; 3017, 3113 (m1-m3); 2707, 2713, 2715, 2825, 3114 (m1-m2); 2712, 2993, 3022 (m1); Dn esq: UFRB-PV 2872, 2997 (m1-m3); 2709, 2716, 2824, 2954, 3018 (m1-m2); 949, 2705, 2711, 2718, 2869, 2876 (m1). Cerradomys sp.: Cr: UFRB-PV 2861 (M1-M3 dir e M1-M3 esq); Mx dir: UFRB-PV 2820 (M1-M3); Mx esq: UFRB-PV 959, 2818, 2990 (M1-M3); 2987 (M1-M2); Dn dir: UFRB-PV 3019, 3020, 3028 (m1); Dn esq: UFRB-PV 2934 (m1-m2). Holochilus sciureus: Cr: UFRB-PV 2901, 2919, 2969 (M1 dir e M1-M3 esq); Mx esq: UFRB-PV 2911, 2918, 2965 (M1-M3); 2992, 3123 (M1-M2); 962, 2988 (M2-M3); 2864, 2989 (M1); 972 (M2); Dn dir: UFRB-PV 907, 2978 (m1-m3); 2852, 2972, 2977 (m1-m2); 936 (m1); Dn esq: UFRB-PV 2956 (m1-m3); 908 (m1-m2), 948 (m2). Necromys lasiurus: Cr: UFRB-PV 2975 (M1-M3 dir e M1-M2 esq); 2857 (M1 dir e M1-M2 esq), Mx dir: UFRBPV 2733, 2952, 2986, 3012 (M1-M2); Mx esq: UFRB-PV 2748, 2822 (M1-M3); 2737, 2740, 2743, 3131 (M1-M2); 2747, 2953, 2985 (M1); Dn dir: UFRB-PV 947, 2829, 2999, 3109, 3112 (m1-m2); 2998 (m1); Dn esq: UFRB-PV 3001, 3002, 3026 (m1-m3); 2731, 2833, 3031 (m1-m2); 2936, 3030, 3119 (m1). Oligoryzomys sp.: Mx esq: UFRB-PV 2735 (M1-M2). Wiedomys pyrrhorhinos: Cr: UFRB-PV 2855, 2858, 2898 (M1-M3 dir e M1-M3 esq); 2859 (M1-M3 dir e M2-M3 esq); 2897 (M1-M3 dir e M1 e M3 esq); 2957 (M1-M2 dir e M1-M2 esq); 906 (M3 dir e M1-M3 esq); Mx dir: UFRB-PV 2819, 3108, 3134, 3176 (M1-M3); Mx esq: UFRB-PV 2823, 3121, 3130 (M1-M3); 956 (M2-M3), 973, 2983 (M1); Dn dir: UFRBPV 2826, 3023 (m1-m3); 3006 (m1-m2); Dn esq: UFRB-PV 2690 (m1-m3); 3021, 3024 (m1-m2); 2710, 2877 (m1).

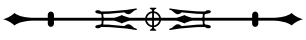

